\title{
Correlation between Internet addiction, depression, anxiety and stress among undergraduate medical students in Azad Kashmir
}

\author{
Arslaan Javaeed ${ }^{1}$, Maria Bint Zafar², \\ Madiha Iqbal ${ }^{3}$, Sanniya Khan Ghauri ${ }^{4}$
}

\begin{abstract}
Objective: To find out the correlation between internet addiction and depression, anxiety and stress among undergraduate medical students in Azad Kashmir.

Methods: A cross-sectional study including 210 undergraduate medical students (first to the fifth year) was done in Poonch Medical College, Azad Kashmir. The data collection tools were DASS21 questionnaire and Young's internet addiction questionnaire. Spearman rank correlation test was done to see the correlation between internet addiction and depression, anxiety, and stress. Data were analyzed by SPSS v23 at a 95\% confidence interval.

Results: A very high prevalence (52.4\%) of moderate to extremely severe internet addiction was observed among the respondents. The mild positive correlation between internet addiction and depression was identified $(p<0.001)$ and similar type of correlation was observed between internet addiction and stress (p 0.003). However, anxiety and internet addiction were not significantly correlated. The prevalence of anxiety and depression among the males were higher than the females, whilst the stress level was almost the same across gender.

Conclusion: Internet addiction has been found to be associated with various psychiatric diseases. In this study, we also observed such correlation. We have also observed a very high level of internet addiction among medical students. The prevalence of internet addiction may further increase in the coming years as the internet will become more cheap, available and include more high quality psychologically addictive contents.
\end{abstract}

KEYWORDS: Internet addiction, Medical Students, DASS21, Anxiety, Depression, Stress.

doi: https://doi.org/10.12669/pjms.35.2.169

How to cite this:

Javaeed A, Zafar MB, Iqbal M, Ghauri SK. Correlation between Internet addiction, depression, anxiety and stress among undergraduate medical students in Azad Kashmir. Pak J Med Sci. 2019;35(2):506-509. doi: https://doi.org/10.12669/pjms.35.2.169

This is an Open Access article distributed under the terms of the Creative Commons Attribution License (http://creativecommons.org/licenses/by/3.0), which permits unrestricted use, distribution, and reproduction in any medium, provided the original work is properly cited.

Arslaan Javaeed, MBBS, M.Phil

2. Maria Bint Zafar, MBBS.

3. Madiha lqbal, MBBS.

4. Sanniya Khan Ghauri, MBBS, MRCEM. Department of Emergency Medicine, Shifa International Hospital, Islamabad, Pakistan. 1,3: Poonch Medical College,

Rawalakot, Azad Kashmir, Pakistan.

Correspondence:

Dr. Sanniya Khan Ghauri, MBBS, MCEM (UK).

Registrar,

Department of Emergency Med.

Shifa International Hospital,

Islamabad, Pakistan.

E-mail: sanniyaghauri89@gmail.com

* Received for Publication:

December 9, 2018

* Corrected and Edited:

* Accepted for Publication:
January 8, 2019

February 12, 2019

\section{INTRODUCTION}

Over the last fifteen years, the number of internet users increased by one thousand fold. The addictive internet usage also proliferated significantly in the last decade. And it has been found to be associated with younger age internet users. ${ }^{1}$ Although internet addiction (IA) is not included in the Diagnostic and Statistical Manual of Mental Health Disorder, Fifth Edition, its association was identified with various psychiatric conditions. ${ }^{2}$ People who spend more than 38 hours a week online, are considered to have an Internet addiction. Internet addiction does not involve the use of an intoxicating drug, 
but it is very similar to pathological gambling in terms of brain biochemistry. ${ }^{3}$ Internet addicts tend to become less responsible for their actions and suffer from social isolation. ${ }^{4}$

Internet is an essential part of life especially for the medical students due to its need for education, research, social networking, and information sharing, banking, shopping etc. ${ }^{5,6}$ Medical students fall in the vulnerable group due to their age, need for internet use and stressful medical training. ${ }^{7,8}$ A comorbid psychopathology systematic review in excessive internet use revealed that $75 \%$ of the examined studies reported a significant correlation between pathological internet use and depression. ${ }^{9}$ A previous study showed the significant relationship between IA and psychiatric symptoms such as depression, obsessive compulsion, interpersonal sensitivity, anxiety, hostility, phobic anxiety, paranoid ideation, and psychoticism. With longer use, more psychiatric symptoms occur. ${ }^{10}$ This study was aimed to assess the correlation between internet addiction and depression, anxiety, and stress among the medical students of Azad Kashmir.

\section{METHODS}

The cross-sectional study was conducted at Poonch Medical College, Azad Kashmir including 210 undergraduate medical students. The medical students were from first to fifth years. Study duration was from April to October 2018. The data were collected using interviewer-administered, well-known DASS21 questionnaire and Young's internet addiction questionnaire. The DASS21 questionnaire has been successfully used across the world in many previous studies to measure depression, anxiety, and stress. ${ }^{11-13}$ The DASS21 questionnaire previously showed a high level of internal consistency (Cronbach's alpha $=0.70$ and above).${ }^{14}$ The DASS21 questionnaire contains 21 4-points Likert scale questions, of which 7 for measuring depression, seven for anxiety, and the remaining seven questions for identifying stress. The Young's internet addiction questionnaire includes twenty Likert scale questions, each of which measures internet addiction at 0 to 6 points scale. ${ }^{15}$ After the extensive literature search, the prevalence of internet addiction was observed to be from $2 \%$ to $7.9 \% .^{16-18}$ Due to variation of prevalence rate in the literature we have assumed a high prevalence rate of $15 \%$ to make sure the sample size was adequate. By applying the popular formulae $\mathrm{N}=\mathrm{Z}^{2 *} \mathrm{P}(1-\mathrm{P}) / \mathrm{e}^{2}$, we have obtained a sample size of 196 . List of all medical students was collected from the database of the medical college. From there 250 medical students were randomly selected using a random number generator of SPSS v23. The interviewer himself approached all 250 medical students with the DASS21 questionnaire. Total 210 medical students gave the consent and completed the questionnaire (Response rate of $84 \%$ ). These 210 medical students were included in the study. Ethical approval was obtained from the ethical review board of Poonch Medical College.

Descriptive statistics were used to present the data in tables and charts. Cronbach's alpha test was done to check the internal consistency of DASS21 and Young's IA questionnaires. Spearman rank correlation test was done to correlate the level of internet addiction and depression, anxiety, and stress levels. The analysis was performed in 95\% confidence interval using the Statistical Package for Social Science (SPSS), version 23.0 (IBM, Armonk, NY, USA).

\section{RESULTS}

Among the 210 respondents, 75 (35.7\%) were male. Baseline characteristics are presented in Table-I. The Chronbach's alpha value of DASS21 questionnaire and Young's IA questionnaire for the current study was 0.820 and 0.852 . According to DASS21 score calculation, extremely severe depression, anxiety, and stress were observed among $40(19.0 \%), 97(46.2 \%)$ and $5(2.4 \%)$ medical students respectively. (Table-II) Only 7 (3.3\%) medical students did not have any internet addiction. The prevalence of moderate to severe internet addiction among the medical students was $52.4 \%$. (Chart-1). All three of severe internet addicts were females. Extremely severe depression was higher among the male respondents $21(28 \%)$

Table-I: Baseline characteristics of the respondents.

\begin{tabular}{lc}
\hline Characteristics & $N(\%)$ \\
\hline Age in years (mean \pm SD) & $21.84 \pm 1.78$ \\
Gender & $75(35.7)$ \\
Male & $135(64.3)$ \\
Female & \\
Year of study & $36(17.1)$ \\
First year & $43(20.5)$ \\
Second year & $38(18.1)$ \\
Third year & $46(21.9)$ \\
Fourth year & $47(22.4)$ \\
Fifth year &
\end{tabular}


Table-II: Depression, anxiety and stress levels of the respondents according to DASS21 scores.

\begin{tabular}{lccc}
\hline Category & $\begin{array}{c}\text { Depression } \\
N(\%)\end{array}$ & $\begin{array}{c}\text { Anxiety } \\
N(\%)\end{array}$ & $\begin{array}{c}\text { Stress } \\
N(\%)\end{array}$ \\
\hline Normal & $66(31.4)$ & $32(15.2)$ & $88(41.9)$ \\
Mild & $25(11.9)$ & $4(1.9)$ & $47(22.4)$ \\
Moderate & $42(20.0)$ & $45(21.4)$ & $33(15.7)$ \\
Severe & $37(17.6)$ & $32(15.2)$ & $37(17.6)$ \\
Extremely severe & $40(19.0)$ & $97(46.2)$ & $5(2.4)$ \\
\hline
\end{tabular}

compared to females 19 (14.1\%). Anxiety was also observed more among the males $69(92.0 \%)$ than females $109(80.7 \%)$. Moderate to severe internet addiction was observed almost equally in males and females, around 36\%.

There was statistically significant mild uphill correlation between depression and internet addiction (correlation coefficient 0.329 and $p$ $<0.001$ ), and an even milder uphill correlation between stress and internet addiction (correlation coefficient .202, p .003). However, anxiety was not significantly correlated with internet addiction $(\mathrm{p}$ 0.391). (Table-III).

\section{DISCUSSION}

Psychological stress on the medical students is relatively higher than other disciplines. A multitude of factors may be responsible for these high stress level. ${ }^{19}$ It is very difficult to know what percentage of the stress comes from internet addiction alone. The global prevalence of internet addiction was $6.0 \% .{ }^{17}$ In contrast, we have found $96.3 \%$ of the medical students to have some degree (mild to extremely severe) of internet addiction. A study done in Saudi Arabia revealed a very high level of depression, anxiety, and stress among the medical students. But the stress level may change depending on the propinquity of examination dates. ${ }^{20}$

An Iranian study showed a higher prevalence of IA among the females which goes against the current study findings as here we have observed a similar level of internet addiction across the gender. ${ }^{21}$

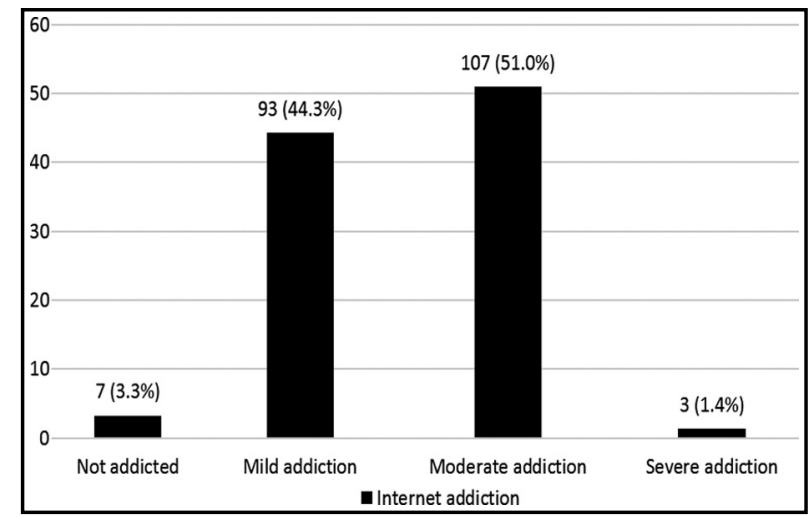

Fig.1: Frequencies and percentages of respondents with different degree of internet addiction.

The significant difference in the prevalence of IA and its relationship with depression, anxiety, and stress were observed in the literature. This might be because of IA is not constant in all time. The internet is becoming more cheap, available and including more services as time progresses. A study done on college students in India revealed a positive correlation between IA and depression, anxiety, and stress. ${ }^{22}$ However, in this study, we did not find any significant correlation between IA and anxiety although the prevalence of moderate to extremely severe anxiety was very high, $82.8 \%$.

\section{CONCLUSION}

Internet addiction has already been associated with a multitude of psychiatric disorders. We have observed a significant relationship between internet addiction and anxiety and stress. The prevalence of internet addiction and its association with psychosocial problems has the potential to increase in future as the availability of internet in general and enrichment of the virtual world with more high-quality, attractive and addictive digital contents are rising.

Limitations of the Study: Being a single centered study, it may not include the representative samples which make the generalization of the study finding appalling. The psychological stress level of the medical student may be subject to change depending on many other factors. The study did not involve any confounders.

Table-III: Correlation between internet addiction and depression, anxiety, and stress.

\begin{tabular}{llccc}
\hline & & Depression & Anxiety & Stress \\
\hline \multirow{3}{*}{ Internet addiction } & Correlation coefficient & 0.329 & 0.060 & 0.202 \\
& p-value & $<0.001$ & 0.391 & 0.003 \\
\hline
\end{tabular}


Acknowledgements: Thanks are due to Prof. Dr. Shabbir Ahmed (Principal Poonch Medical College), and administrative staff especially Waseem Azam, Tayyab Ali, Asad Sharif and Javed Yousuf for supporting research activities.

\section{Grant Support E Financial Disclosures: None.}

Source of funding: Self-funded.

Conflict of Interest: None.

\section{REFERENCES}

1. Kuss DJ, Lopez-Fernandez O. Internet addiction and problematic Internet use: A systematic review of clinical research. World J Psychiat. 2016;6(1):143. doi: 10.5498/wjp. v6.i1.143

2. Ko CH, Yen JY, Yen CF, Chen CS, Chen CC. The association between Internet addiction and psychiatric disorder: a review of the literature. Eur Psychiat. 2012;27(1):1-8 doi: 10.1016/j.eurpsy.2010.04.011

3. Alavi SS, Maracy MR, Jannatifard F, Eslami M. The effect of psychiatric symptoms on the internet addiction disorder in Isfahan's University students. Journal Resource Medical Science: Off J Isfahan Uni Med Sci. 2011;16(6):793.

4. Moeedfar S, Habbibpour Getabi K, Ganjee A. Study of Internet addiction between adolescent \& young 15-25 years in Tehran University. Glob Med J Tehran Uni. 2007;2(4):5579.

5. Gedam SR, Ghosh S, Modi L, Goyal A, Mansharamani H. Study of internet addiction: Prevalence, pattern, and psychopathology among health professional undergraduates. Ind J Soc Psychiat. 2017;33(4):305. doi: 10.4103/ijsp.ijsp_70_16

6. Chaudhari B, Menon $\mathrm{P}$, Saldanha D, Tewari A Bhattacharya L. Internet addiction and its determinants among medical students. Indust Psychiat J. 2015;24(2):158. doi: 10.4103/0972-6748.181729

7. Ranganatha SC, Usha S. Prevalence and pattern of internet addiction among medical students, Bengaluru. Int J Commun Med Pub Health. 2017;4(12):4680-4684. doi: 10.18203/2394-6040.ijcmph20175350

8. Wolf TM. Stress, coping and health: enhancing well-being during medical school. Med Educ. 1994;28(1):8-17. doi: 10.1111/j.1365-2923.1994.tb02679.x

9. Carli V, Durkee T, Wasserman D, Hadlaczky G, Despalins $\mathrm{R}$, Kramarz E, et al. The association between pathological internet use and comorbid psychopathology: a systematic review. Psychopathol.2013;46(1):1-3. doi:10.1159/000337971

10. Mustafa KO. Internet addiction and psychopatology. TOJET: Turk Onli J Edu Tech. 2011;10(1).

11. Osman A, Wong JL, Bagge CL, Freedenthal S, Gutierrez PM, Lozano G. The depression anxiety stress Scales - 21 (DASS 21): further examination of dimensions, scale reliability, and correlates. J Clin Psychol. 2012;68(12):1322-1338. doi: 10.1002/jclp.21908
12. Sinclair SJ, Siefert CJ, Slavin-Mulford JM, Stein MB, Renna M, Blais MA. Psychometric evaluation and normative data for the depression, anxiety, and stress scales-21 (DASS-21) in a nonclinical sample of US adults. Evaluat Health Prof. 2012;35(3):259-279. doi: $10.1177 / 0163278711424282$

13. Oei TP, Sawang S, Goh YW, Mukhtar F. Using the depression anxiety stress scale 21 (DASS-21) across cultures. Int J Psychol. 2013;48(6):1018-1029. doi: 10.1080/00207594.2012.755535

14. Tran TD, Tran T, Fisher J. Validation of the depression anxiety stress scales (DASS) 21 as a screening instrument for depression and anxiety in a rural community-based cohort of northern Vietnamese women. BMC Psychiat. 2013;13(1):24. doi: 10.1186/1471-244X-13-24

15. Young KS. Internet addiction: The emergence of a new clinical disorder. Cyberpsychol Behav. 1998;1(3):237-244. doi: 10.1089/cpb.1998.1.237

16. Liu $X$, Bao Z, Wang Z. Internet use and internet addiction disorder among medical students: A case from China. Asian Soc Sci. 2009;6(1):28. doi:10.5539/ass.v6n1p28

17. Cheng $\mathrm{C}, \mathrm{Li} \mathrm{AY}$. Internet addiction prevalence and quality of (real) life: A meta-analysis of 31 nations across seven world regions. Cyberpsychol Behav Soc Net. 2014;17(12):755-760. doi: 10.1089 /cyber.2014.0317

18. Koyuncu T, Unsal A, Arslantas D. Assessment of internet addiction and loneliness in secondary and high school students. J Pak Med Assoc. 2014;64(9):998-1002.

19. Yusoff MS, Yaacob MJ, Naing NN. Psychometric properties of the Medical Student Well-Being Index among medical students in a Malaysian medical school. Asian J Psychiat. 2013;6(1):60-65. doi: 10.1016/j.ajp.2012.09.001

20. Kulsoom B, Afsar NA. Stress anxiety and depression among medical students in a multiethnic setting. Neuropsychiat Disease Treatment. 2015;11:1713. doi: 10.2147/NDT.S83577.

21. Gholamian B, Shahnazi H, Hassanzadeh A. The prevalence of internet addiction and its association with depression, anxiety, and stress, among high-school students. Int J Pediatr. 2017;5(4):4763-4770. doi: 10.22038/ ijp.2017.22516.1883

22. Panicker JA, Sachdev R. Relations among loneliness, depression, anxiety, stress and problematic internet use. Int J Resin Appl Natural Soc Sci. 2014;2(9):1-10.

\section{Authors Contribution:}

AJ, SKG: Conceived, designed and did statistical analysis \& editing of manuscript.

MBZ, MI: Did data collection and manuscript writing.

AJ: Did review and final approval of manuscript. 